\title{
The influence of cognitive load and walking speed on gait regularity in children and young adults
}

\author{
Sabine Schaefer ${ }^{*}$, Danilo Jagenow ${ }^{1}$, Julius Verrel, Ulman Lindenberger \\ Center for Lifespan Psychology, Max Planck Institute for Human Development, Berlin, Germany
}

\section{A R T I C L E I N F O}

\section{Article history:}

Received 14 July 2014

Received in revised form 19 September 2014

Accepted 14 October 2014

\section{Keywords:}

Dual-task

Walking

Cognition

Children

Young adults

\begin{abstract}
A B S T R A C T
The dual-process account of sensorimotor-cognitive interactions postulates that easy cognitive tasks can lead to performance improvements in the motor domain (e.g., an increased stability while walking or balancing) across the lifespan. However, cross-domain resource competition can lead to performance decrements in motor tasks when the concurrent cognitive task is very difficult, and older adults have shown performance decrements in their motor functioning under such circumstances. Resource limitations are particularly pronounced not only in old adulthood, but also in childhood. The current study investigates the relationship of walking speed and cognitive load on walking regularity in 7-and 9 -year olds and young adults, with 18 participants in each group. Participants were walking on a treadmill at their preferred speed, and with speeds that were 30\% faster and 30\% slower than preferred. Regularity of lower-body coordination was operationalized as the residual variance of principal component analyses performed on the data of a motion analysis system. All age groups showed a more regular gait with increasing walking speed. Young adults' gait regularity was not influenced by cognitive load, whereas children showed a U-shaped relationship of cognitive load and walking regularity, with the highest regularity when performing an easy cognitive task. It can be concluded that children are also influenced by cross-domain resources competition in challenging cognitive-motor dual-task situations. (c) 2014 Elsevier B.V. All rights reserved.
\end{abstract}

\section{Introduction}

Cognitive-motor dual-task situations are very common in everyday life, for example when talking on a mobile phone while crossing a busy street. Although walking is a fairly automatized task, walking patterns might change under cognitive load. Several studies have shown that attentional processes are involved in the regulation of gait [1-7]. In addition, the interference of a cognitive and a motor task may be more pronounced in children and old adults, given that walking may be less automatized due to lack of experience/immaturity (children) or declining sensorimotor function (older adults), such that more attention needs to be invested into this task domain.

Gait changes can be observed during childhood, with older children walking with more consistent, straighter, longer and narrower steps [8]. Furthermore, the interindividual and

\footnotetext{
* Corresponding author at: Center for Lifespan Psychology, Max Planck Institute for Human Development, Lentzeallee 94, D-14195 Berlin, Germany. Tel.: +493082406425.

E-mail address: schaefer@mpib-berlin.mpg.de (S. Schaefer).

1 Now at the Department of Education and Psychology, Free University Berlin, Germany.
}

intraindividual variability of walking decreases with age [911]. Hausdorff et al. [12] investigated 50 healthy children 3-14 years of age and found that stride-to-stride variations in gait cycle duration decrease with age. Mature stride dynamics were not completely developed in 7-year-old children, whereas 11year-olds showed adult-like levels of gait variability. Other studies also converge on the finding that typical adult walking patterns show up at ages between 7 and 12 years [13-15].

Kinematic walking data are highly structured, showing relatively invariant patterns of spatiotemporal correlation between movements of different body parts [16]. Principal component analysis (PCA) allows splitting multivariate data into a main (regular) portion and a residual (irregular) portion. Applied to gait data, the amount of residual variance is a measure of the irregularity of the walking pattern $[17,18]$. Thus, higher values indicate decreased gait regularity. By taking into account timecontinuous information and defining irregularity as deviation from an individualized invariant multivariate pattern, this is likely a more sensitive measure of gait regularity than discrete measures based on step pattern variability (cf. 18). Note that, similar to measures based on step variability, gait regularity is not necessarily directly linked to biomechanical or dynamic stability of the walking pattern, but less stable walking likely results in a 
less regular walking pattern. Since children show a more regular gait with advancing age, PCA identifies temporal characteristics of kinematic curves that discriminate between age groups [9].

A previous study by Schaefer et al. [19] asked 9-year olds and young adults to walk on a treadmill at their preferred speed and at a fixed, slower-than-preferred speed, either under single-task conditions or when performing a working memory task. Participants in both age groups improved their cognitive performance when walking at their preferred speed as opposed to sitting or walking at the fixed speed. Stride-length and stride-time variability tended to decrease with cognitive load in young adults, whereas children showed an increase in walking variability when cognitive load was very high. Such a U-shaped function connecting motor performances to different cognitive loads had previously been shown in old adults when balancing [20] or when walking on a treadmill [21], and has been interpreted as an instance of the dual-process account relating cognitive control to motor control performance. While an external focus of attention initially improves walking regularity or balance for all participants, motor performances of older adults and children tend to deteriorate when the cognitive load is very high.

The present study aims to investigate the influence of cognitive load and walking speed on walking patterns in children and young adults, using the residual variance from a PCA for assessing gait characteristics on a treadmill. 7-year olds and 9-year olds were chosen since gait regularity [9-15] and cognitive performances [22] continue to improve in that age range. The study design includes a preferred speed condition as well as walking speeds that are $30 \%$ slower and 30\% faster than preferred. We predicted that walking regularity will show the U-shaped function in children with easier cognitive tasks asserting a stabilizing effect on walking, while demanding cognitive tasks lead to decreased regularity again. Young adults' walking was not expected to suffer from cognitive load. The study also investigates whether the cognitive performance improvements found by Schaefer et al. can be replicated and extended to faster-than-preferred walking speeds, and whether an increased gait regularity induced by faster walking speeds reduces the influence of cognitive load.

\section{Method}

\subsection{Participants}

We recruited 7-year-old children $(M=7.63$; $S D=0.31)$, 9-yearold children $(M=9.48 ; S D=0.29)$ and young adults (20-30 years; $M=26.57 ; S D=1.77)$, with 18 participants and equal numbers of males and females in each age group.

Participants were drawn from the institute's participant pool and were excluded if they had a history of diagnosed disorders directly affecting balance and gait, neurologic disorders, or attention-deficit-hyperactivity syndrome. All participants had normal or corrected-to-normal vision and normal hearing, and showed performances in standardized tests on cognitive speed, short-term memory and word knowledge that are consistent with the developmental literature [22].

The experiment consisted of four sessions each lasting about $1 \mathrm{~h}$. Participants received $€ 40$ for their participation. All participants (for children their legal guardian) signed a written consent form prior to testing. The study was approved by the institute's ethics committee.

\subsection{Apparatus}

Treadmill walking was performed on a Woodway treadmill (Woodway GmbH, Weil am Rhein, Germany) with a $200 \mathrm{~cm}$ long $\times 70 \mathrm{~cm}$ wide surface on ground level. Participants were secured with a safety belt. A virtual path was projected on a $270 \mathrm{~cm} \times 202 \mathrm{~cm}$ screen in front of the treadmill, whose visual flow was synchronized to each participant's walking speed.

Participants were wearing standardized sport shoes. For optical motion analysis, sixteen $14 \mathrm{~mm}$ diameter retro-reflective markers were attached to relevant anatomical landmarks according to the Vicon Plug-In-Gait model, with 4 markers placed on the pelvis and 6 on each leg and foot.

A Vicon MX motion analysis system with 10 near infrared cameras (Vicon Motion Systems Ltd, Oxford, UK) was used for tracking the position of the markers, with a temporal resolution of 200 frames per second. After manual pre-processing, motion data were analyzed with the Vicon Nexus software (v.1.4.116) and custom-written MATLAB scripts (MathWorks, Natick, USA).

\subsection{Experimental tasks}

\subsubsection{Cognitive task}

An N-back trial of the current study consisted of a sequence of 40 auditorily presented digits ranging from " 1 " to " 9 ", drawn randomly for presentation, with the constraint that digits would never appear in a naturally ascending sequence (i.e., 1, 2, 3, 4). Depending on the difficulty level, participants are asked to judge whether a new item is identical to the item two (2-back) or three (3-back) positions back in the sequence [23]. If a target is detected, participants are asked to say "Tap". All trials included seven targets. To prevent rhythmical influences on gait, the stimuli were administered with an interstimulus interval jittered between 2000 and $2500 \mathrm{~ms}$. The instruction emphasized accuracy over response speed. Each correct response to a target scored one point; for a false alarm one point was subtracted from the score (maximum score $=7$ points). Performance feedback was given after each trial.

\subsubsection{Walking}

The motor task consisted of walking on a motor-driven treadmill in three different speeds. The preferred speed was instructed as "allowing participants a natural and rhythmic walking, which they feel comfortable with". It was chosen individually for each participant. The two additional conditions were $30 \%$-faster-than-preferred or 30\%-slower-than-preferred. In each session, participants were given 5 min to familiarize with the respective speed before motion-capture was started.

For the PCA, gait data from each trial and participant kinematic data from all markers were processed and analyzed separately. Data were presented as Cartesian coordinates in an array of $3000 \times 48$ dimensions. The amount of variance explained by the first three principal components was $M=96.38 \%(S D=.23)$, with the fourth component explaining less than $1 \%$ of variance, which is in line with previous findings (e.g., 18). The cut-off between the regular and the irregular patterns was therefore set after the third principal component.

\subsection{Procedure}

Four testing sessions were distributed across a time period of up to 2 weeks, with at least 1 day of rest between individual testing sessions. Fig. 1 presents a diagram outlining the experimental procedures. Participants were tested individually by two experimenters.

Sessions 2, 3 and 4 used one of the three walking speeds, whose order was counterbalanced across participants. To avoid articulation influences on the walking parameters, the time window for motion capture did not include any $\mathrm{N}$-back targets [24]. This time window consisted of 11 digits (20 s), and it was randomly set after 10 or 20 digits to avoid positional predictability. Single-task 


\begin{tabular}{|c|}
\hline Session 1: \\
N-back instruction \\
Determination of preferred speed on the treadmill \\
\hline Sessions 2, 3, and 4: \\
Single task block 1: \\
2-back while sitting (1 trial) \\
3-back while sitting (1 trial) \\
Walking only (1 trial) \\
Dual-task block: \\
2-back while walking (2 trials) \\
3-back while walking (2 trials) \\
Single task block 2: \\
Walking only (1 trial) \\
2-back while sitting (1 trial) \\
3-back while sitting (1 trial)
\end{tabular}

Fig. 1. Flowchart of the experimental procedure. In sessions 2, 3 and 4, walking speeds (slow, preferred, fast) remained constant throughout the respective session. The order of these walking speeds was counterbalanced across participants.

walking trials lasted $60 \mathrm{~s}$, but only a 15-s interval in the middle of the trial was analyzed. For dual-task trials, no task-priority instructions were given.

Mixed-design ANOVAs were performed separately for the dependent variables ( $\mathrm{N}$-back scores, residual variance of PCA), with cognitive load (2: 2-back, 3-back) and walking speed (4: no walking, preferred, 30\% slower, $30 \%$ faster) as within-subjects factors, and age group (3: 7-year olds, 9-year olds, young adults) as between-subjects factor. For within-subjects effects, the multivariate $F$ values are reported, along with partial Eta square values for effect sizes. Significant interactions were followed up by $t$-tests. The alpha level was .05 (Bonferroni corrected to .025 for follow-up analyses).

\section{Results}

\subsection{Cognitive performance}

An overall ANOVA with walking speed (4: no walking, slow, preferred, fast) and cognitive load (2: 2-back, 3-back) and as within-subjects factors and age group [3] as between-subjects factor was conducted. Fig. 2 depicts the results graphically, and Table 1 summarizes the statistics.

Neither walking as such nor walking speed influenced cognitive performance (no main effect of walking speed). The main effect of age group was significant, with the lowest N-back scores for 7-year olds and the highest scores for young adults. Walking speed did not interact with age group. A significant main effect for cognitive load was detected, which was qualified by an interaction of cognitive load and age group. In addition, the interaction of cognitive load and walking speed reached significance, whereas the triple interaction of walking speed, cognitive load and age failed to reach significance.

To follow up the significant interaction of cognitive load and age group, N-back scores were aggregated over walking speed, and univariate ANOVAs with age group [3] as the independent factor were conducted for 2-back and 3-back conditions separately. For 2-back, there was a significant effect of age, $F(2,51)=14.03$, $p<.001, \eta_{p}^{2}=.355$. The Tukey-test for multiple comparisons revealed that 7-year olds differed from 9 -year olds, $p=.002$, and 7 -years olds differed from young adults, $p<.001$. However, the difference between 9-years olds and young adults did not reach significance, $p=.277$. For 3-back, there was a significant effect of age, $F(2,51)=16.21$, $p<.001, \eta_{p}^{2}=.389$, and the respective Tukey-test revealed that each age group differed significantly from each other age group (7-year old vs. 9-year olds, $p=.023 ; 7$-years olds vs. young adults, $p<.001 ; 9$-years olds vs. young adults, $p=.013$ ).

The interaction of cognitive load and walking speed was followed up by independent samples $t$-tests comparing 2-back to 3-back for each of the four speed conditions separately, averaging across the three age groups. In all four analyses, $2-$ back performances were significantly better than 3-back performances (sitting: $t$ $(53)=13.90, p<.001$; slow walking: $t(53)=12.34, p<.001$; preferred speed walking: $t(53)=11.76, p<.001$; fast walking: $t(53)=9.26, p<.001$ ).

\subsection{Walking}

Concerning the preferred walking speeds that were chosen by participants, young adults $(M=3.50 \mathrm{~km} / \mathrm{h}, S D=.47)$ walked faster than 9-year olds $(M=2.96$, $S D=.48), t(34)=3.44, p<.001$, and 7-year olds $(M=2.81, S D=.33)$ did not differ from 9-year olds, $t(34)=1.09, p=.283$.

An overall ANOVA with cognitive load (3: no load, 2-back, 3-back) and walking speed (3: slow, preferred, fast) as within-subjects factors and age group [3] as between-subjects factor was conducted, with the residual variance of the PCA as the dependent variable. Fig. 3 depicts the results graphically, and Table 2 summarizes the statistics. A significant main effect of cognitive load was detected, which was qualified by an interaction of cognitive load and age group. The main effect of walking speed was significant, reflecting a decrease of gait variability with increasing velocity, which interacted with age group as well.

Neither the interaction of cognitive load and walking speed, nor the three-way interaction of cognitive load, walking speed and age reached significance. Furthermore, statistics revealed a significant main effect of age group, reflecting the highest gait variability in 7-year-old children and the lowest variability in young adults.

To follow up on the interactions of cognitive load and age group and of walking speed and age group, three repeated-measures ANOVAs were calculated, one for each age group, with the within-subjects factors cognitive load [3] and walking

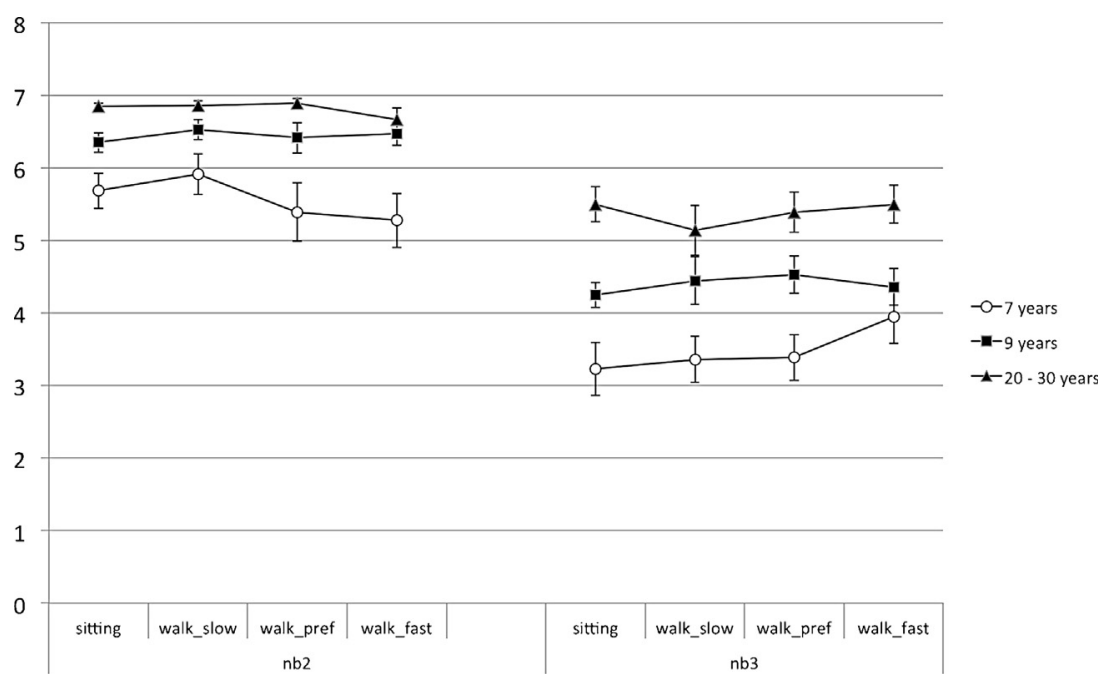

Fig. 2. $N$-back performance for all age groups under all task conditions. Error bars = SE mean. 
Table 1

Results of the mixed-design ANOVA for cognition.

\begin{tabular}{|c|c|c|c|c|}
\hline Source & $d f$ & $F$ & $\eta_{p}^{2}$ & $p$ \\
\hline \multicolumn{5}{|l|}{ Between subjects } \\
\hline Age & 2 & 17.82 & .41 & $<.001$ \\
\hline Age within-group error & 51 & $(5.04)$ & & \\
\hline \multicolumn{5}{|l|}{ Within subjects } \\
\hline Walking speed & 3 & .13 & .00 & .941 \\
\hline Walking speed $\times$ age & 6 & .59 & .02 & .740 \\
\hline Walking speed $\times$ age within-group error & 153 & $(.76)$ & & \\
\hline Cognitive load & 1 & 344.49 & .87 & $<.001$ \\
\hline Cognitive load $\times$ age & 2 & 4.44 & .15 & .017 \\
\hline Cognitive load $\times$ age within-group error & 51 & $(1.08)$ & & \\
\hline Walking speed $\times$ cognitive load & 3 & 3.49 & .06 & .017 \\
\hline Walking speed $\times$ cognitive load $\times$ age & 6 & 1.75 & .06 & .113 \\
\hline $\begin{array}{l}\text { Walking speed } \times \text { cognitive load } \times \text { age } \\
\text { within-group error }\end{array}$ & 153 & $(.48)$ & & \\
\hline
\end{tabular}

Note: Values enclosed in parentheses represent mean square errors. speed [3]. For 7-year olds, a main effect of cognitive load was found, and the quadratic trend for this effect reached significance $(p=.005)$, indicating that walking variability decreased from no load to 2-back, but increased again for 3-back. A similar pattern occurred for 9-year olds, with the main effect of cognitive load reaching significance, as well as both the quadratic $(p<.001)$ and the linear $(p=.004)$ trend. In young adults, however, cognitive load did not reach significance. Gait regularity is systematically influenced by cognitive load in children, but not in young adults. All three groups show a more regular gait when walking faster.

\section{Discussion}

The present study investigated how gait is influenced by cognitive load and walking speed in children and young adults. Walking regularity was assessed by the residual variance, that is, the amount of variance not explained by the invariant portion (first three principal components) of the multivariate lower-body gait pattern. By taking into account time-continuous information and individual movement patterns, this measure of regularity is likely

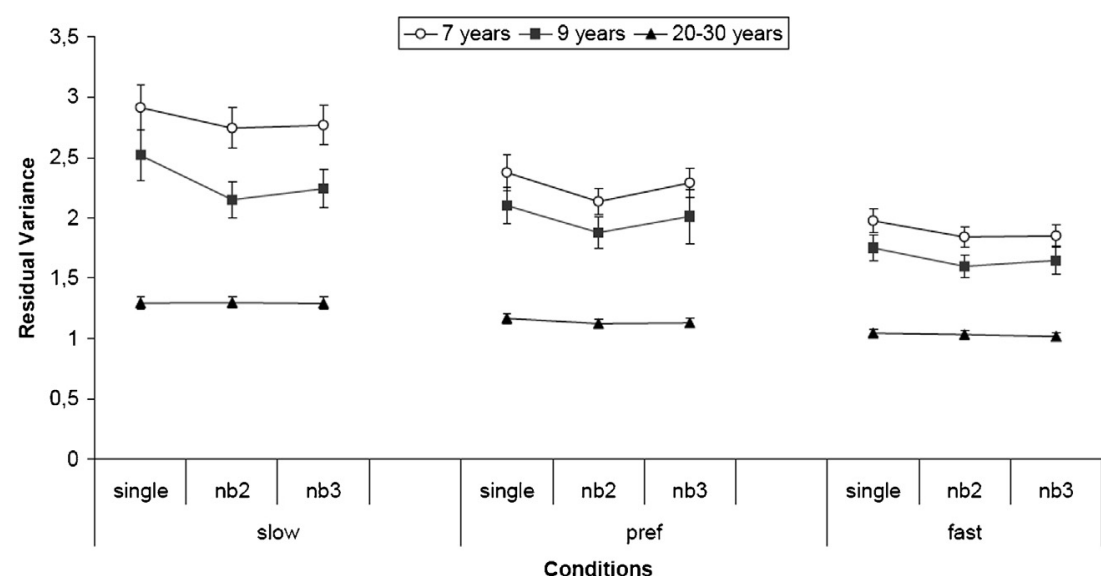

Fig. 3. Walking regularity (residual variance of PCA) for all age groups under all task conditions. Error bars = SE mean.

Table 2

Results of the mixed-design ANOVA and of the three follow-up ANOVAs for gait.

\begin{tabular}{|c|c|c|c|c|c|}
\hline Type of analysis & Source & $d f$ & $F$ & $\eta_{p}^{2}$ & $p$ \\
\hline \multicolumn{6}{|l|}{ Between subjects } \\
\hline \multirow[t]{2}{*}{ Overall mixed-design ANOVA } & Age & 2 & 37.20 & .59 & $<.001$ \\
\hline & Age within-group error & 51 & $(1.57)$ & & \\
\hline \multicolumn{6}{|l|}{ Within subjects } \\
\hline & Walking speed & 2 & 80.08 & .61 & $<.001$ \\
\hline & Walking speed $\times$ age & 4 & 8.08 & .24 & $<.001$ \\
\hline & Walking speed $\times$ age within-group error & 102 & $(.19)$ & & \\
\hline & Cognitive load & 2 & 22.06 & .30 & $<.001$ \\
\hline & Cognitive load $\times$ age & 4 & 4.60 & .15 & .002 \\
\hline & Cognitive load $\times$ age within-group error & 102 & $(.04)$ & & \\
\hline & Walking speed $\times$ cognitive load & 4 & 1.13 & .02 & .344 \\
\hline & Walking speed $\times$ cognitive load $\times$ age & 8 & .76 & .03 & 640 \\
\hline & Walking speed $\times$ cognitive load $\times$ age within-group error & 204 & $(.04)$ & & \\
\hline \multirow[t]{4}{*}{ Follow-up ANOVA, 7-year olds } & Walking Speed & 2 & 32.61 & .66 & $<.001$ \\
\hline & Cognitive Load & 2 & 6.68 & .28 & .004 \\
\hline & Walking speed $\times$ cognitive load & 4 & .47 & .03 & .759 \\
\hline & Walking speed $\times$ cognitive load within-group error & 68 & $(.06)$ & & \\
\hline \multirow[t]{4}{*}{ Follow-up ANOVA, 9-year olds } & Walking speed & 2 & 29.60 & .64 & $<.001$ \\
\hline & Cognitive load & 2 & 15.83 & .48 & $<.001$ \\
\hline & Walking speed $\times$ cognitive load & 4 & 1.35 & .07 & .261 \\
\hline & Walking speed $\times$ cognitive load within-group error & 68 & $(.05)$ & & \\
\hline \multirow[t]{4}{*}{ Follow-up ANOVA, young adults } & Walking speed & & 45.07 & .73 & $<.001$ \\
\hline & Cognitive load & 2 & 1.86 & .09 & .171 \\
\hline & Walking speed $\times$ cognitive load & 4 & 1.43 & .07 & .235 \\
\hline & Walking speed $\times$ cognitive load within-group error & 68 & $(.00)$ & & \\
\hline
\end{tabular}

Note: Values enclosed in parentheses represent mean square errors. 
more sensitive to subtle changes in motor performance due to dual-task-interference than other measures of gait variability [18].

Increases in walking speed led to a more regular gait in all age groups. Jordan et al. [25] also found decreasing stride-to-stride fluctuations with increasing walking velocity in young females. A study by Abernethy et al. [26] had young adults walk and run at different speeds on a treadmill while performing an auditory reaction-time task. When participants were instructed to maintain very fast walking speeds that were normally associated with running, they showed costs in the cognitive task. In the current study, however, even the $30 \%$ faster than preferred speed was too slow to elicit running throughout the study.

Cognitive load did not influence walking regularity in young adults. Children, however, demonstrated a U-shaped relationship, with increased gait regularity when working on an easier cognitive task as compared to no task, and decreased regularity when cognitive load was high. This pattern had already been shown in old adults in the domain of balance [20] as well as walking $[18,21]$. The authors of these studies argue that the stabilization with easy cognitive tasks can be attributed to the effect of internal versus external focus of attention, since focusing on the movement under single-task conditions might disrupt the self-organizing dynamics of the motor system $[27,28]$, whereas an easy cognitive task enables the system to smoothly execute the automatized motor task. However, a dual-task situation also requires sharing resources between the two tasks, and children and old adults have to deal with limited resources in cognitive and motor domains. A rather demanding cognitive task therefore leads to performance decrements (i.e., reduced regularity) in the motor domain in these groups. Future work with this paradigm should also investigate the influence of very easy cognitive tasks (like 1-back) on children's walking patterns. As opposed to the study by Verrel et al. [18], young adults in the current study did not show increased gait regularity with increasing cognitive load. Since 3-back, the most difficult condition of the current study, was easier for young adults than for children, it remains on open question whether young adults would decrease their gait regularity when working on a very difficult cognitive task.

Schaefer et al. [19] reported cognitive performance improvements in children and young adults when walking on a treadmill with their preferred speed. This effect was not replicated in the current study. All age groups showed comparable cognitive performances while sitting or while walking with the three different speeds. The study by Schaefer et al. used four different difficulty levels of the cognitive task (1-back to 4-back), whereas the current study only used 2-back and 3-back. It is possible that the inclusion of a more difficult version of N-back would have led to performance improvements while walking, since the Schaefer et al. paper reports larger improvements with increasing cognitive load. Future research should investigate which specific exercise conditions are necessary to produce $\mathrm{N}$-back performance improvements, whether these improvements can be found in N-back response speed as well, and in how far they generalize to other cognitive tasks.

To conclude, cognitive performances in the current study remained stable across different motor tasks: It did not matter whether participants were sitting or walking on a treadmill with different speeds. Furthermore, young adults did not show any changes in their walking regularity with increasing cognitive load. Children showed a U-shaped relationship between cognitive load and walking regularity, indicating that the dual-process account of sensorimotor-cognitive interactions can be generalized to children, including walking speeds that are slower or faster than one's preferred speed.

\section{Acknowledgements}

These studies were carried out at and funded by the Max Planck Institute for Human Development in Berlin. We thank Michael
Schellenbach and Paul Czienskowski for programming the experimental tasks and Djamila Maleika for helpful discussions. We also would like to thank Gabi Faust, Larissa Arndt, Miriam Balt, Sandra Kutnick, Nina Lisofsky, Antoinette von Schönau, and Astrid Strey for their help with data collection.

\section{Conflict of interest}

The authors declare no conflict of interest.

\section{References}

[1] Lajoie Y, Teasdale N, Bard C. Attentional demands for static and dynamic equilibrium. Exp Brain Res 1993;97:139-44.

[2] Li KZH, Lindenberger U, Freund AM, Baltes PB. Walking while memorizing: age-related differences in compensatory behavior. Psychol Sci 2001;12:230-7.

[3] Lindenberger U, Marsiske M, Baltes PB. Memorizing while walking: increase in dual-task costs from young adulthood to old age. Psychol Aging 2000;15: 417-36.

[4] Woollacott M, Shumway-Cook A. Attention and the control of posture and gait: a review of an emerging area of research. Gait Posture 2002;16:1-14.

[5] Huang H-J, Mercer VS. Dual-task methodology: applications in studies of cognitive and motor performance in adults and children. Pediatr Phys Ther 2001; 13:133-40.

[6] Hung Y-C, Meredith G, Gill S. Influence of dual task constraints during walking for children. Gait Posture 2013;38:450-4

[7] Hung Y-C, Gill S, Meredith G. Influence of dual-task constraints on whole-body organization during walking in children who are overweight and obese. Am J Phys Med Rehabil 2013;92:461-71.

[8] Adolph KA, Vereijken B, Shrout PE. What changes in infant walking and why Child Dev 2003;74:475-97.

[9] Chester VL, Wringley AT. The identification of age-related differences in kinetic gait parameters using principal component analysis. Clin Biomech $2008 ; 212-20$

[10] Dusing SC, Thorpe DE. A normative sample of temporal and spatial gait parameters in children using the GAITRite electronic walkway. Gait Posture 2007;25:135-9

[11] Sutherland D. The development of mature gait. Gait Posture 1997;6:163-70.

[12] Hausdorff JM, Zemany L, Peng C-K, Goldberger AL. Maturation of gait dynamics: stride-to-stride variability and its temporal organization in children. J Appl Physiol 1999;86:1040-7.

[13] Jeng SF, Liao HF, Lai JS, Hou JW. Optimization of walking in children. Med Sci Sports Exerc 1997;29:370-6.

[14] Norlin R, Odenrick P, Sandlund B. Development of gait in the normal child. J Pediatr Orthoped 1981;1:261-6.

[15] Sutherland D, Olshen R, Cooper L, Woo L. The development of mature gait. J Bone Joint Surg 1980;62:336-53.

[16] Daffertshofer A, Lamoth CJ, Meijer OG, Beek PJ. PCA in studying coordination and variability: a tutorial. Clin Biomech 2004;19:415-28.

[17] Deluzio KJ, Wyss UP, Zee B, Costigan PA, Sorbie C. Principal component models of knee kinematics and kinetics: normal versus pathological gait patterns. Human Movement Sci 1997;16:201-17.

[18] Verrel J, Lövdén M, Schellenbach M, Schaefer S, Lindenberger U. Interacting effects of cognitive load and adult age on the regularity of whole-body motion during treadmill walking. Psychol Aging 2009;24:75-81.

[19] Schaefer S, Lövdén M, Wieckhorst B, Lindenberger U. Cognitive performance is improved while walking: differences in cognitive-sensorimotor couplings between children and young adults. Eur J Dev Psychol 2010;7:371-89.

[20] Huxhold O, Li S-C, Schmiedek F, Lindenberger U. Dual-tasking postural control: aging and the effects of cognitive demand in conjunction with focus of attention. Brain Res Bull 2006;69:294-305.

[21] Lövdén M, Schaefer S, Pohlmeyer AE, Lindenberger U. Walking variability and working-memory load in aging: a dual-process account relating cognitive control to motor control performance. J Gerontol: Psychol Sci 2008;63B: P121-8.

[22] Li S-C, Lindenberger U, Hommel B, Aschersleben G, Prinz W, Baltes PB. Transformations in the couplings among intellectual abilities and constituent cognitive processes across the life span. Psychol Sci 2004;15:155-63.

[23] Dobbs AR, Rule BG. Adult age differences in working memory. Psychol Aging 1989;4:500-3.

[24] Dault MC, Yardley L, Frank JS. Does articulation contribute to modifications of postural control during dual-task paradigms. Cogn Brain Res 2003;16:434-40.

[25] Jordan K, Challis JH, Newell KM. Walking speed influences on gait cycle variability. Gait Posture 2007;26:128-34.

[26] Abernethy B, Hanna A, Plooy A. The attentional demands of preferred and nonpreferred gait patterns. Gait Posture 2002;15:256-65.

[27] Beilock SL, Bertenthal BI, McCoy AM, Carr TH. Haste does not always make waste: expertise, direction of attention, and speed versus accuracy in performing sensorimotor skills. Psychonom Bull Rev 2004:11:373-9.

[28] Wulf G, Prinz W. Directing attention to movement effects enhances learning: a review. Res Q Exerc Sport 2001;78:384-9. 